\title{
APROXIMACIÓN EPISTEMOLOGICA A LAS RELACIONES ENTRE LA DIDÁCTICA DE LAS CIENCIAS NATURALES Y LA DIDÁCTICA GENERAL
}

\author{
María Laura Eder y Agustín Adúriz Bravo*
}

\begin{abstract}
Both within didactics of science (science education) and within general educational studies, there is a large arnount of literature concerning the episternological status of these disciplines. One of the rnain epistemological issues is that focussing on the relationships between didactics of science and general didactics (theory of teaching). In this paper we attempt to inspect such relationships through the following axes that we consider of interest: the concept of discipline and the ways in which this term is used; views on science; a comparíson between natural and social sciences; pedagogy and social science; and the reationships between content-specific and non-specific didactics. Sorne hypotheses referring to didactics of science are proposed.
\end{abstract}

\section{RESUMEN}

Tanto en la didáctica de las ciencias naturales como en las distintas ramas de la pedagogía hay una importante producción académica ocupada del análisis epistemológico interno de estas disciplinas. Una de las cuestiones epistemológicas más relevantes es la que hace a las relaciones entre la didáctica específica de las ciencias y la didáctica general. En este trabajo se hace un intento de explorar estas relaciones planteando algunos eles teóricos que se consideran de interés: el concepto de disciplina y los usos que se dan a este término; las visiones sobre la ciencia; las diferencias y semejanzas entre las ciencias naturales y sociales; la inclusión de la didáctica entre las ciencias sociales; y las relaciones entre didáctica general y didácticas especificas. Se plantean algunas hipótesis referidas específicamente a la didáctica de las ciencias.

\section{INTRODUCCIÓN}

Entre las grandes cuestiones teóricas que se plantean hoy en el campo de as didácticas, nos interesa particularmente aquella que se refiere a la relación entre la didáctica general y las didácticas especificas, con especial referencia a la didáctica de las ciencias naturales. Este interés puede inscribirse en una serie de estudios de naturaleza epistemológica que se desarrollan tanto en la didáctica general (Camilloni, 1994; Camilloni et al., 1996) como en la didáctica de las ciencias ${ }^{1}$ (Adúriz-Bravo, 1999; Eder, 1998; Jiménez Aleixandre, 1988; Porlán, 1992, 1998). Como señala Rivera Medero (1997), esta reflexión metateórica en las distintas disciplinas sociales se constituye en una necesidad.

\footnotetext{
* Centro de Formación e Investigación en Enseñanza de las Ciencias. Facultad de Ciencias Exactas y Naturales. Universidad de Buenos Aires, aula 14, pabellón 2, Ciudad Universitaria. (1428) Buenos Aires, Argentina, e-mail (primer autor): mleder @filo.uba.ar

${ }^{1}$ La denominación abreviada de didáctica de las ciencias se refiere a la didáctica de las ciencias naturales o experimentales. Los investigadores en esta área — que suelen ser científicos provenientes de la biología, la tísica, La química, etc-, utilizan dicha denominación sin aclarar de qué ciencias se trata.
} 
En este trabajo nos proponemos iniciar un análisis epistemológico de las relaciones entre la didáctica de las ciencias y la didáctica general, a partir de una afirmación realizada por Rafael Porlán (1992) en su artículo "La didáctica de las ciencias. Una disciplina emergente", en el que traza el itinerario de este campo y enumera los aspectos que, a su juicio, deberían desarrollarse para su definitiva consolidación disciplinar. Dice Porlán:

Consideramos a la Didáctica de las Ciencias, pues, como una disciplina emergente que forma parte de la didáctica y que se incluye en el campo más amplio de las Ciencias de la Educación. Aun cuando su origen está muy vinculado a las ciencias experimentales, actualmente, después de un largo proceso de reflexión y reelaboración epistemológica, psicológica y didáctica, está plenamente integrada en las ciencias sociales. En gran medida, este proceso de redefinición, que ha estado inmerso en un cambio mucho más amplio y profundo de carácter social, económico, político y filosófico, ha marcado la evolución de sus cuarenta años de existencia más o menos formal. (Porlán, 1992: 70)

A partir del planteo que realiza Porlán, se podría pensar en una serie de inclusiones sucesivas: la didáctica de las ciencias en el campo de la didáctica, esta en el de las ciencias de la educación, y estas últimas entre las ciencias sociales (o culturales, o humanas). Resulta de interés plantear algunas de las implicaciones que tiene, en el análisis del proceso de construcción del conocimiento, la delimitación de un campo en el que confluyen desde diferentes lugares las ciencias sociales y las ciencias naturales.

Para desarrollar este planteo abordaremos las siguientes cuestiones: el concepto de disciplina y los diversos usos que se dan a este término; las concepciones de ciencia; las particularidades de las ciencias naturales y sociales, así como sus semejanzas; la inclusión de la didáctica entre las ciencias sociales; las relaciones entre didáctica general y didácticas específicas; y por último, ya en el ámbito de las conclusiones, algunas hipótesis que surgen a partir de los aspectos antes considerados, referidas específicamente a la didáctica de las ciencias.

\section{LA DIDÁCTICA COMO DISCIPLINA}

Al decir de Díaz Barriga (1997), la didáctica como disciplina ha estado tradicionalmente vinculada a visiones de tipo instrumentalista (tecnologizantes), fundadas epistemológicamente en el positivismo y en el funcionalismo. Hoy la didáctica puede ser considerada una disciplina teórica, histórica y política:

Es teórica en cuanto responde a concepciones amplias de la educación (y esto la engarzaría a una teoría de la educación), de la sociedad, del sujeto, etc. Es histórica en cuanto sus propuestas son resultados de momentos históricos específicos. (...) Es política porque su propuesta se engarza en un proyecto social. (Díaz Barriga, 1997: 23)

Ahora bien, ¿por qué hablamos de disciplina y no dé ciencia? En los planteos que, desde lugares diferentes, realizan Rafael Porlán (didácta de las ciencias español) y Ángel Díaz Barriga (pedagogo mexicano), nos encontramos con el mismo término de disciplina. Es conveniente, entonces, tratar de comprender no sólo qué designa, sino también por qué se lo utiliza. Creemos que ello marca una determinada postura epistemológica.

Podríamos pensar la disciplina como un dominio más o menos sistemático del conocimiento, referido a ciertos objetos de estudio, que implica métodos de indagación y 
validación del conocimiento particulares, ciertas actitudes y valores, y una comunidad científica que la lleva adelante. En este sentido, las disciplinas estarían relacionadas con las especialidades científicas, tecnológicas, artísticas y humanísticas (Adúriz-Bravo, 1999).

Algunos autores (Apostel et al., 1977) plantean sin embargo que uno de los usos más habituales del término disciplina es el de sustituir la palabra ciencia, aunque el término disciplina conlleva la noción adicional de enseñar una ciencia (Eder, 1998). Este matiz tiene importancia para nuestra discusión. Vemos entonces que muchas veces se utilizan de modo casi intercambiable ambos conceptos.

La pedagoga argentina Mariana Maggio analiza así la noción de disciplina:

Precisamente, la noción "disciplina" empieza a destacarse en el movimiento denominado conceptual empirista, donde pueden identificarse, entre otros, los aportes de Schwab, Phenix y Bruner. Para Phenix (...) las disciplinas son aquellos campos de conocimiento con una estructura peculiar que se pone de manifiesto a través de sus realizaciones (es decir, de los resultados de la investigación) y que agrupa en torno suyo a una comunidad especializada. Las disciplinas abarcan todas las formas de conocimiento posible y además proporcionan los modelos de "desarrollo cognoscitivo potencial". Aparecen como base objetiva para la selección del currículum por poseer "estructuras de crecimiento naturales que le son inherentes', esto es, no sujetas a decisiones subjetivas o históricamente condicionadas. (Maggio, 1998:27 y 28)

Nuevamente observamos aquí la vinculación estrecha entre disciplina y enseñanza, que también se evidencia en la etimología de la palabra (relacionada con discipulus, alumno: ver Eder, 1998). Cabría plantearse si, en el discurso pedagógico actual, se reconoce esta importante característica en el término disciplina, o simplemente su utilización soslaya la dificultad de incluir la didáctica dentro de alguna ciencia, por lo cual se la describe mejor como una disciplina a la que sólo algunos autores califican de "científica".

Ciertamente, para muchos, disciplina asume el significado muy general que le otorgan Gardner y Boix-Mansilla (1994), lo que permite eludir la discusión acerca de la cientificidad de este campo de conocimiento que es la didáctica:

Las disciplinas constituyen los modos más sofisticados desarrollados hasta ahora para pensar e investigar temas, que durante mucho tiempo han fascinado y asombrado a los individuos reflexivos (...), representan los modos principales para que los individuos trasciendan la ignorancia (...), se convierten — cuando se usan en forma relevante- en nuestra lente más aguda para mirar el mundo.

Las disciplinas tienden en si mismas a diferentes tipos de roles y performances (...). Distintas disciplinas requieren de diferentes estilos analíticos, diferentes aproximaciones a la resolución de problemas y descubrimientos, distintas disposiciones e inteligencias. (pp. 3 y 4 )

\section{LA DIDÁCTICA COMO DISCIPLINA CIENTÍFICA}

Analicemos ahora el adjetivo con que muchas veces se califica a la disciplina: ¿qué hace que una disciplina sea científica? Aún hoy persiste en el sentido común y en la 
denominada epistemología espontánea de los docentes gran parte del programa mecanicista que supuso el afincamiento del determinismo más radical en la ciencia:

El conocimiento objetivo y verdadero estaba al alcance del método científico y el conseguirlo sólo era cuestión de tiempo; la verdad, como tal, no podía tener matices, cuando se llegaba a ella a través de una correcta descripción matemática se había logrado el conocimiento científico. Lo mismo sucedía con el error, simplemente se consideraba que un conocimiento erróneo no era científico. La verdad de una ley iluminaba la predicción a través de los datos iniciales; en teoría, era posible determinar con rigor absoluto el curso de los acontecimientos. (Luffiego of al., 1994: 89)

Señala Carlos Hoyos Medina (1997) que el término ciencia generalmente se difunde, en la actualidad, en este sentido funcionalista heredado del positivismo:

Proveniente de la jerga anglosajona, science alude a sistemas de comprobación por medio de metódicas racionales, lógicamente establecidas. Estas han de operar sobre su objeto, en contrastación empírica observacional, de manera que, estableciendo su regularidad empírica, se pueda inferir su media universal. Todo con plena exclusión del sujeto cognoscente. La ciencia, su método, parecen avanzar por si mismos. Sólo basta cumplir con el requisito de constituirse una proposición que alcance la categoría de enunciado de base, lo demás estará sujeto a la lógica misma del o los modelos ad hoc para su tratamiento (científico). (pp. 30 y 31; subrayado en el original)

Las ciencias sociales, según este autor, han sido condicionadas a construir sus objetos bajo el crisol de la experiencia empírica, para de ahí poder derivar el significado, ya sea por verificación (según el positivismo lógico) ${ }^{2}$, o por falsación (según el racionalismo crítico) $)^{3}$.

Por su parte, Alicia Camilloni (1994) plantea que:

Según ese concepto positivista de la ciencia, ella tiene como meta alcanzar la verdad, y postula como ideal el logro de un conocimiento absoluto de la realidad en sí misma. Pretende llegar a conocer la totalidad de su dominio. Y, aunque algunos (...) autores acepten, incluso, que las teorías científicas tienen un carácter hipotético, metas móviles, un camino itinerante y carencial, se descartan siempre de las teorías que son calificadas como científicas todas las implicaciones normativas. En esta concepción, la ciencia es eminentemente desinteresada, en el mismo sentido en que Aristóteles diferenciaba la teoría de la praxis y de la poiesis. (p. 28; subrayado en el original)

Hoy, las nuevas visiones acerca del conocimiento científico caracterizan de manera diferente esta problemática. Para Brown (1984), por ejemplo:

\footnotetext{
2 "Era posible demarcar sin ambigüedad el conocimiento científico de otros tipos de conocimiento. Partiendo de proposiciones elementales significativas, o lo que es lo mismo, de proposiciones que hacen referencia a hechos simples y que pueden ser verificadas empíricamente, sería posible construir leyes y teorías científicas mediante combinación lógica de estas proposiciones elementales. De esta manera podría obtenerse un conocimiento probado, es decir, verdadero y objetivo y, por lo tanto, universal. La demarcación entre ciencia y seudociencia consistiría precisamente en exigir que las teorías fueran probadas y verificadas a través de un estudio positivista" (Luffiego et al., 1994: 90).

${ }^{3}$ Popper considera que "la ciencia es simplemente asunto detener ideas y ponerlas a prueba, una y otra vez, intentando siempre demostrar que las ideas están equivocadas, para así aprender de nuestros errores” (Pérez Tamayo, 1993:173).
} 
Lo que constituye el nervio de la ciencia es la investigación en curso, antes que los resultados establecidos. La ciencia consiste en una serie de proyectos de investigación estructurados mediante las presuposiciones aceptadas que determinan qué observaciones se han de hacer, cómo se han de interpretar, qué fenómenos son problemáticos y cómo han de ser tratados estos problemas. Cuando cambian las presuposiciones de una disciplina científica, quedan transformadas también tanto la estructura de esa disciplina como la imagen de la realidad del científico. El único aspecto permanente de la ciencia es la investigación. (pp. 221 y 222)

Y Larry Laudan (1986) plantea que:

[N]o hay una fundamental diferencia de tipo entre la ciencia y otras formas de indagación intelectual. Todo pretende dotar de sentido al mundo y a nuestra experiencia. Todas las teorías tanto científicas como de otro tipo, están igualmente sujetas a compromisos empíricos y conceptuales. Las disciplinas que denominamos "ciencias" son, generalmente, más progresivas que las "no ciencias"; ciertamente, pudiera ser que las llamemos "ciencias" simplemente porque son más progresivas, más que a causa de algún rasgo metodológico o sustantivo que tengan en común. De ser así, las diferencias que hay resultan ser de grado más que de tipo. (pp. 21 y 22)

Estos análisis permiten considerar a la didáctica general como una disciplina caracterizada por su progresividad y por su capacidad para resolver problemas, superando en cierto modo el debate acerca de su carácter científico. Le cabe entonces a la didáctica no ya la discusión sobre su cientificidad, sino la vigilancia epistemológica que le permita reconocer aquellas producciones que son fruto de investigaciones rigurosas (más allá de la utilización de metodologías diferentes) y también aquellas líneas o programas de investigación que son más progresivos, en términos de Laudan, dentro de la disciplina.

En tal sentido, la aceptación de un estatuto de cientificidad para las didácticas y otras disciplinas sociales nos plantea un problema de reconstrucción del modelo de ciencia canónico. Esta reconstrucción ya está esbozada en la nueva filosofía de la ciencia de los años ' 60 y '70, cuyos autores hemos mencionado más arriba, pero puede profundizarse actualmente por medio de los llamados modelos cognitivos de ciencia (Giere, 1992; Adúriz-Bravo, 1999).

\section{CIENCIAS SOCIALES Y CIENCIAS NATURALES}

Dado que el objetivo de nuestro trabajo requiere ubicar elementos que caracterizan a las ciencias sociales y a las naturales, resulta de interés realizar algunos señalamientos acerca de sus semejanzas y diferencias. Haremos un breve recorrido por algunas de las posiciones que las revisan.

Por una parte, nos encontramos con teóricos de distintas extracciones que plantean algunos puntos de similitud. En un trabajo sobre la epistemología de las ciencias humanas, Jean Piaget (1972) toma dos aspectos sobre los que considera que no pueden oponerse las ciencias naturales de las ciencias del hombre: método y campo. Así:

[En relación a los métodos,] parece imposible introducir una oposición entre las ciencias del hombre y las ciencias naturales, ni desde el punto de vista de la experimentación, ni desde el del cálculo o el de la deducción. En el caso de las 
ciencias naturales, la experimentación es general, salvo en dos tipos notables de excepción: el de las matemáticas o ciencias derivadas (mecánica relacional y física matemática, por oposición a teórica), porque se trata de disciplinas deductivas, y el de la astronomía y el de la geología, porque la escala de los fenómenos con respecto al observador, prohíbe que éste modifique aquéllos. Sin embargo, en el caso de las ciencias del hombre, la experimentación es más limitada que en las ciencias naturales, pero debido a las mismas razones. Allí donde ella es posible, como en psicología y en fonética experimentales, o en cibernética (en la cual un modelo material tan pronto puede fracasar como resultar exitoso), obedece a las mismas reglas que en biología, para dar un ejemplo (...). En los casos en que la experimentación no interviene, es porque se trata de disciplinas deductivas, como la lógica, o porque la escala de los fenómenos impide su manipulación (sociología, lingüística, buena parte de la economía, la demografía, etc). (pp. 183 y 184)

Nos interesa recuperar esta reflexión, a pesar de las objeciones que pueden hacérsele (Eder, 1998), para mostrar como, desde posiciones epistemológicas diferentes, aparece la preocupación por acercar las distintas ciencias. Por otra parte, en relación a los campos que abarcan las ciencias del hombre, Piaget continúa:

El hecho notable que se opone a toda separación radical con las ciencias naturales radica en que no hay una sola de aquéllas que no termine por extenderse hasta el terreno de éstas, en tanto que las generalizaciones de las segundas interesan cada vez más a las primeras. (p. 185).

Karl Popper (1973), por su parte, caracteriza su teoría acerca de la unidad del método en las ciencias del siguiente modo:

a) El método de las Ciencias Sociales, al igual que el de las Ciencias de la Naturaleza, radica en ensayar posibles soluciones para sus problemas (y Se proponen y critican soluciones. En el caso de que un ensayo de solución no resulte accesible a la crítica objetiva, es preciso excluirlo por no científico, aunque sólo provisionalmente.

b) Si es accesible a una crítica objetiva, intentamos refutarlo; porque toda crítica consiste en intentos de refutación.

c) Si un ensayo de solución es refutado por nuestra crítica, buscamos otro.

d) Si resiste la crítica, lo aceptamos provisionalmente; y desde luego, lo aceptamos principalmente como digno de seguir siendo discutido y criticado.

e) El método de la ciencia es, pues, el de la tentativa de solución, el del ensayo (o idea) de solución sometido al más estricto control crítico. (...)

f) La llamada objetividad de la ciencia radica en la objetividad del método crítico; lo cual quiere decir, sobre todo, que no hay teoría que esté liberada de la crítica, y que los métodos lógicos de los que se sirve la crítica (...) son objetivos. (p.)

Mary Hesse (1980), epistemóloga británica, plantea cinco aspectos con los que podemos hoy caracterizar simultáneamente a las ciencias naturales y las humanas, desmarcándose así de una posición epistemológica secesionista (Estany, 1993) común en el pasado reciente:

1. Los datos son inseparables de la teoría; lo que cuenta como datos es determinado a la luz de alguna interpretación teórica, y los hechos empíricos están reconstruidos en el seno de la teoría (Izquierdo y Adúriz-Bravo, en prensa).

2. Las teorías son formas paradigrnáticas de "ver" los hechos. 
3. Las relaciones nomológicas en los datos empíricos son internas; lo que cuenta como hechos está constituido por lo que la teoría dice sobre sus interreaciones con otros hechos.

4. El lenguaje de las ciencias naturales es irreductiblemente metafórico e inexacto; es formalizable sólo a costa de una distorsión de la dinámica histórica de la ciencia.

5. El sentido que damos al mundo está determinado por la teoría, y se desprende de la coherencia teórica, más que de la correspondencia con los hechos.

Por otra parte, podría pensarse que en la actualidad se está produciendo un cambio en las ciencias naturales que las aproxima a las ciencias humanas. Quizás sea uno de los factores de este cambio el desarrollo de la llamada teoría del caos. Esta teoría, junto con el principio de indeterminación y la emergente perspectiva de la complejidad, merecen un párrafo especial.

A principios del siglo XIX, el mecanicismo basado en una interpretación estrecha del determinismo newtoniano inició un período de apoteosis y contribuyó a asentar la opinión según la cual "en el universo no ocurría más que el desarrollo inexorable de la causalidad férrea, de modo que la situación en cada momento era la única posible tras la que había en el anterior y daba paso, a su vez, a otra única siguiente" (Fernández Rañada, 1990: 5).

Pero Fernández Rañada plantea tres rupturas de este paradigma mecanicista, que se va sustituyendo:

1. Por el de los sistemas complejos con muchas variables, tantas que resulta imposible seguir la traza de cada una de ellas y hay que recurrir al empleo de promedios y de leyes probabilísticas.

2. Por el de los sistemas cuánticos, como los átomos, moléculas, núcleos o partículas elementales, cuyas leyes son de naturaleza distinta de las de Newton.

3. Por el de los sistemas caóticos, que tienen un comportamiento muy complejo, a pesar de que presentan aspecto simple y pocos grados de libertad; son a la vez deterministas e impredictibles. (1990: 5)

La visión determinista se modificó con la enunciación del principio de indeterminación, por el físico Werner Heisenberg, como ramificación de sus trabajos en mecánica cuántica. Pero además de las consecuencias de este principio, los sistemas dinámicos de comportamiento caótico contribuyen a una nueva visión de 'un mundo probabilista, en la que se imbrican y entreveran cadenas causales deterministas que terminan cuando se destruye totalmente la cantidad de información sobre el estado inicial. (...) De este modo, orden y caos, determinismo y probabilidades se juntan y complementan en un mundo que resulta así más complejo y rico que la visión fría del mecanicismo y cuyo comportamiento se sigue de la acción íntimamente ligada de azar y necesidad" (Fernández Rañada, 1990: 8).

Por su parte, Luffiego et al. (1994) plantean:

En palabras de Prigogine y Stengers (1983): (...) las ciencias de la naturaleza se han liberado de una concepción estrecha de la realidad objetiva, que cree que debe negar en sus principios la novedad y la diversidad en nombre de una ley universal inmutable. 
Se han liberado de una fascinación que no representaba la racionalidad como cerrada, el conocimiento como en vías de terminación. Están, desde ahora, abiertas a lo imprevisto, del cual ya no hacen el signo de un conocimiento imperfecto, de un control insuficiente. Desde ahora, se han abierto al diálogo, con una naturaleza que no puede ser dominada con una mirada teórica, sino solamente explorada, con un mundo abierto al cual pertenecemos, en la construcción del cual participamos (...). Ha llegado el momento de nuevas alianzas, ligadas desde siempre, durante mucho tiempo desconocidas, entre la historia de los hombres, la historia de las sociedades, de sus conocimientos y la aventura exploradora de la naturaleza".

Por estos motivos, la nueva ciencia, la ciencia de la complejidad, deberá poner en marcha una nueva metodología, a la cual Morin (1984) denomina transdisciplinar, que, sin negar las particularidades de las distintas disciplinas, establezca un diálogo entre las mismas y aborde problemáticas con presupuestos epistemológicos menos ambiciosos que los positivistas y con criterios metodológicos holísticos e integradores. (p. 92)

El surgimiento de la teoría del caos y de esta llamada "ciencia de la complejidad" parece abrir nuevas perspectivas para pensar las relaciones entre las ciencias sociales y las ciencias naturales y, por lo tanto, entre la didáctica general y la didáctica de las ciencias naturales. Dentro de estas perspectivas, la disolución parcial de los límites entre las diversas disciplinas enfocadas sobre la enseñanza y sobre las ciencias, y el abordaje de problemas desde ejes transdisciplinares, parecen abrir un campo prometedor para la investigación didáctica.

\section{LA DIDÁCTICA COMO DISCIPLINA SOCIAL}

Se plantea que la didáctica se inscribe en el ámbito de las ciencias sociales ${ }^{4}$ (Camilloni, 1994). Señalemos, entonces, algunos elementos que caracterizan a estas ciencias.

Históricamente las ciencias sociales nacen como tales, aproximadamente en el siglo XVIII, cuando se hace evidente que la sociedad y la vida de los hombres en ésta no evolucionaba tan naturalmente como se pensaba; se da una crisis en la aparente claridad existente en cuanto a las ciencias, dado que existían aquellas que determinaban tajan-tomento normas y procesos metodológicos sobre los estudios de las ciencias naturales, los cuales podrían ser traspolados a las incipientes "ciencias", en tanto que si no eran observadas desde ese marco científico no se podía hablar de ciencia en éstas. (...) [P]ara salvar su estatus de científicas, las ciencias sociales desde un marco positivista estudian sus fenómenos y hechos como cosas naturales, que se pueden estudiar e interpretar y conocer separadas de otros hechos, sin tomar en cuenta que los hechos sociales no son cosas estáticas y naturales sino que éstos son creados por los hombres en las relaciones sociales, en donde lo más difícil de comprobar, en sí, es la objetividad del hecho, dado que cada postura sobre la realidad concibe como objetivo y subjetivo diferentes elementos. (Mata García, 1997: 124 y 126)

\footnotetext{
${ }^{4}$ Algunos autores identifican la didáctica (general) con una ciencia social. Otros (cf. Adúriz Bravo, 1999), hablan de la didáctica como una disciplina científica social, rama de una ciencia, que seria la pedagogía o ciencia de la educación.
} 
1.Popkewitz (1994), por otro lado, plantea desde una mirada histórica el surgimiento de las ciencias sociales en la universidad, para satisfacer la demanda que hacía el estado de conocimientos de los procesos y organizaciones sociales sobre los que podría influir la intervención estatal. Durante la era progresista (nos ubicamos a mediados del siglo XIX, en los EEUU) "el crédito del nuevo experto profesional consistía en un saber basado exclusivamente en las reglas de la lógica y en la referencia a lo empírico, en vez de en la categoría social de quien hablara o en la autoridad de Dios" (p. 94).

Los primeros investigadores que se autodenominaron científicos sociales procedían de diversos medios profesionales y carecían de una formación superior específica o de "métodos" que los apoyasen, aparte de la creencia compartida de que el objetivo de las ciencias sociales consistía en dirigir la mejora social a través de una acción estatal más directa. Así:

Cuando tas Ciencias Sociales se organizaron en torno a asociaciones de disciplinas profesionales, surgieron intensos debates sobre el papel de los científicos sociales en los procesos de mejora social. Algunos vincularon su maestría profesional con la ética social, convencidos de que había que relacionar las obligaciones sociales del cristianismo con las condiciones de la clase trabajadora. (...) Otros científicos sociales (...) abogaban por un enfoque que integrase las cuestiones sociales, económicas y culturales en el estudio de las instituciones. No obstante, con el paso del tiempo, la orientación de corte activista perdió vigencia, quedando en situación marginal. La mayoría de las organizaciones profesionales adoptaron la postura de que los científicos debían ser observadores desinteresados y de que el conocimiento científico era neutral respecto al mundo de la política y de los valores sociales. El saber de la ciencia social sería útil para la acción política, orientando la administración y la evaluación de las acciones sociales, pero no tenía que tomar partido. (Popkewitz, 1994: 102 y 103)

Ubiquémonos ahora desde la perspectiva de Habermas para considerar este punto. Jürgen Habermas (1988) argumenta que diferentes formas de ciencia no sólo emplean distintos modos de razonamiento (como planteaba ya Aristóteles), sino que sirven a diferentes clases de intereses mediante el conocimiento creativo o constitutivo.

Carr (1990) caracteriza así el pensamiento de Habermas:

La ciencia empírico-analítica (...) empleaba la clase de razonamiento técnico identificado por Aristóteles; reconociendo su instrumental, su carácter de fines-medios, describió esta forma de ciencia como guiada por un interés técnico. Las ciencias hermenéuticas, en cambio (...) empleaban lo que Aristóteles describía como modos prácticos de razonamiento; de ahí que Habermas las describiera como guiadas por un interés práctico constitutivo de saber, un interés en guiar, informar y educar a los lectores interpretando el mundo y nuestras maneras de entenderlo, desfilando experiencia y suministrando ejemplos de las consecuencias históricas de actuar de una manera o de otra (correcta o incorrectamente) bajo circunstancias históricas diferentes. (p. 26; subrayado en el original)

Habermas desarrolla además un tercer tipo de interés, el interés emancipatorio. Este es el que caracteriza las acciones y prácticas de los científicos sociales que trabajan en función de la racionalidad, la justicia y la libertad. La ciencia social crítica de Habermas no es entonces simplemente una teoría que aspira a demostrar relaciones entre pensamiento y acción en los individuos, es una clase de teoría social que aspira a la crítica ideológica, 
la organización de la ilustración en grupos sociales y sociedades, y la organización de la acción social y política para mejorar el mundo.

Ahora bien, ¿qué relación existe entre estos tres tipos de ciencia y sus derivaciones? En el caso de las ciencias empírico-analíticas, la posibilidad de su utilización técnica se deduce de las reglas según las cuales aplicamos teorías a la realidad. El interés práctico que guía las ciencias hermenéuticas o interpretativas genera conocimiento en forma de entendimiento interpretativo, capaz de informar y guiar el juicio práctico. La ciencia social crítica, por su parte, "se orienta hacia la capacitación de las personas a través de la retrospección para que se conozcan a sí mismas y a las situaciones por las que discurre su vida y, de esta forma, traer a la conciencia un proceso de formación social que establezca, a su vez, las condiciones en que debe desarrollarse el discurso práctico en el sentido de acción prudente" (Pla i Molins, 1993: 63).

Esta postura nos plantea así un nuevo eje de discusión: no se trata de dicotomizar las ciencias ( $y$, consecuentemente, las prácticas que de ellas se derivan) en sociales y naturales, sino de reconocer el interés constitutivo que subyace a cada ciencia. Una misma ciencia social puede constituirse desde diferentes intereses.

\section{DIDÁCTICA GENERAL Y DIDÁCTICA DE LAS CIENCIAS NATURALES}

Parafraseando a Alicia Camilloni (1994), podemos decir que si la enseñanza de las ciencias naturales se presenta como una labor de extrema complejidad cuando se la encara seriamente, la construcción de una didáctica de las ciencias naturales, esto es, de una teoría de la enseñanza de las ciencias naturales, se revela doblemente intrincada. A la complejidad inicial de su objeto se añade el controvertido carácter epistemológico de la didáctica como disciplina. Se suman a esto la particular situación epistemológica que viven las ciencias naturales, con el surgimiento de numerosos planteos metodológicos nuevos, y los profundos cambios que la educación científica experimenta en los últimos años del siglo (Adúriz Bravo, 1999).

Es importante en este punto abordar brevemente la relación entre la didáctica general y las didácticas específicas. Cristina Davini la analiza recuperando el análisis de Schwab, que si bien tiene ya más de dos décadas, mantiene su vigencia para comprender la evolución actual de la producción en didáctica:

Puede distinguirse una fuga en dos órdenes:

- $\quad$ por un lado, la construcción de toque daremos en llamar "megateorias" en el campo de la didáctica general, comprometidas con un discurso interpretativo que pretende reunir el abanico de producciones científicas en un marco global comprensivo, con la consecuente pérdida de producción de "reglas de acción" (...).

- $\quad$ por el otro, la elaboración de "teorías diafragmáticas" en las didácticas especiales que, focalizando la mirada en una o dos dimensiones, reducen el proceso de enseñanza a una tarea formativa en las distintas materias y se constituyen como feorías autonomizadas y fragmentarias. (Davini, 1996: 54)

Desde esta perspectiva, la didáctica general -tratando de superar el enfoque eficientista tecnocrático-, aumentó su caudal interpretativo-descriptivo, aunque este es 
todavía hoy fragmentario, y dejó el lugar de las propuestas de acción a las didácticas especificas centradas en los diversos contenidos de instrucción. Actualmente, entonces:

Pareciera que de la enseñanza se encargan los especialistas "confenidisfas"

-y no los didactas - amparados en el recorte de dos niveles de la realidad: el contenido y sus reglas y el sujeto del aprendizaje.

Si bien es cierto y legítimo plantear que para las especializaciones de la didáctica se requiere el trabajo conjunto y solidario con los expertos de las distintas disciplinas tanto para la delimitación epistemológica del objeto como para su tratamiento en la enseñanza-, el problema se plantea cuando las propuestas se generan atomizadas de un proyecto didáctico global para la escuela 0 , en otros términos, cuando en lugar de un trabajo conjunto se opera la "colonización" de un experto sobre el otro. (Davini, 1996: 53)

Es importante señalar, en relación a los aportes de las didácticas específicas, que muchas propuestas de enseñanza que de ellas surgen terminan por trascender las disciplinas particulares en las que se gestaron. Las ideas centrales que subyacen y que han sido rastreadas pueden intercambiarse entre las diferentes áreas sin perder potencialidad. Esto supondría, en un futuro muy próximo, producciones significativas generalizables. Esta situación de transferencia nos permite hablar de una reconstitución de la didáctica general a partir de la diferentes didácticas específicas, pero en un nivel epistémico superior (Adúniz-Bravo, 1999). Podemos ejemplificar este planteo enunciando conceptos surgidos de la didáctica de la matemática y de la de las ciencias que hoy tienen valor para comprender el proceso de enseñanza en otras áreas: transposición didáctica, ciencia escolar, contrato didáctico, comunidades de práctica, concepciones alternativas.

Las propuestas didácticas específicas "representan productos de un "diálogo" entre los especialistas en los distintos campos y los aportes de corrientes actuales de la psicología cognitiva", entre ellos, los derivados de las obras de Piaget, Bruner, Vigotsky y Ausubel. Así, "la hegemonía de la psicología sigue presente, sólo que articulada al aprendizaje de un campo determinado de conocimientos" (Davini, 1996: 63 y 64).

Desde la didáctica de las ciencias podemos recuperar algunos aspectos que hacen a la historia de la disciplina, comentados con la visión de sus propios miembros:

El final de la década de los '70 y el comienzo de los '80 estuvo marcado por una explosión de iniciativas para cambiar la escuela; en ciencias se insistía en la importancia de las actividades prácticas, en el estudio del entorno y en la adquisición de destrezas científicas (...). En los casos en los que existía una fundamentación teórica, ésta era de carácter inductivista, considerando la observación, independientemente de los modelos o hipótesis, como punto de partida de los métodos de trabajo de la ciencia. (...) Otra característica del trabajo de innovación en esa etapa, aún no superada del todo en la actualidad, era su carácter no acumulativo (...). Esto significa que, contradiciendo el tipo de avance que se produce en un campo de conocimientos, en que las nuevas aportaciones se basan en las anteriores - sea para confirmarlas o para refutarlas - aquí cada nueva actividad (...) parecía hacer volver al punto cero. Las consecuencias fueron una atomización de los debates y una circularidad en las propuestas, dando la impresión de que no se avanzaba. (Jiménez Aleixandre, 1988: 8 y 9) 
Rafael Porlán (1992), en el artículo anteriormente citado, plantea la influencia determinante que tuvieron, en el desarrollo de la didáctica de las ciencias, las aportaciones de la llamada nueva filosofía de la ciencia. Señala también un importante cambio que se ha producido en los últimos años en este campo de conocimiento:

La Didáctica de las Ciencias ha sufrido un importante proceso de clarificación. Ha pasado de ser un conjunto de prescripciones curriculares de carácter tecnológico, que pretendía trasladar a la escuela una lógica positivista de la ciencia y de su método, sin tener en cuenta, para ello, el conjunto de variables mediadoras que existen en los sistemas de enseñanza-aprendizaje, a ser una disciplina posible (...), de carácter práctico y complejo, que dispone ya, aunque de manera rudimentaria, de algunos de los requisitos imprescindibles para ser considerada como tal (...): una ubicación epistemológica más clara, una comunidad investigadora incipiente, unos medios de comunicación y contraste (revistas, congresos, etc), un cuerpo de conocimientos específicos, así como un objeto de estudio y una problemática reconocida por dicha comunidad. (p. 69; subrayado en el original)

Además añade:

El campo de estudio de la Didáctica de las Ciencias está constituido por el conjunto de los contextos (sistemas) de enseñanza-aprendizaje institucionalizados, en la medida en que manejan información relacionada con los sistemas naturales. Esto quiere decir que los problemas que sean relevantes para la didáctica lo son también para la Didáctica de las Ciencias, y viceversa. (p. 70; el subrayado es nuestro)

Para finalizar este apartado, resulta de importancia señalar cuáles son las líneas de investigación actuales (Porlán, 1992, 1993; Gil-Pérez, 1994; Adúriz Bravo, 1999), que apuntan a tres campos principales:

1. El conocimiento científico (contenido central del aprendizaje académico): en lo que respecta a sus aspectos históricos y epistemológicos (concepción de ciencia).

2. El conocimiento cotidiano (ámbito desde el que los profesores y estudiantes interactúan entre sí y con los contenidos): conocimientos previos de los alumnos (cuáles son, su origen, cómo se modifican).

3. La relación entre ambos: cómo aprenden los alumnos (así como para el avance de las ciencias se hace necesario un cambio no sólo conceptual sino también metodológico, en el aprendizaje de los alumnos es necesario plantear otros modos de construir el conocimiento). Se incorpora el estudio de la resolución de problemas como instrumento de familiarización con las estrategias del trabajo científico.

Un rápido análisis nos permite plantear que las líneas de investigación que se están llevando adelante en la didáctica de las ciencias naturales parecen estar más vinculadas a las áreas de la psicología educativa y de la epistemología que al objeto propio de la didáctica, la enseñanza. Sin embargo, en la última década se verifica una creciente integración de la perspectiva pedagógica a las otras dos (Adúriz-Bravo, 1999).

\section{A MODO DE CONCLUSIÓN}


Revisando todo lo planteado en los diferentes apartados, surgen algunas conclusiones con las que, a modo de hipótesis de trabajo, cerramos este análisis:

1. La didáctica es una disciplina que integra una naturaleza explicativa con una normativa; esto no obtura su carácter progresivo y su capacidad para resolver problemas. Como disciplina científica debe plantearse qué tipo de intereses guían su construcción y, al ocuparse de la enseñanza del campo particular de las ciencias naturales, analizar si la concepción de ciencia que subyace a ambos grupos de ciencias es coherente. Y como disciplina tecnológica debe analizar la naturaleza de sus prescripciones y estudiar los modos de operar con ellas en la formación del docente como profesional.

2. Si, como dice Rafael Porlán, "los problemas que sean relevantes para la didáctica lo son también para la Didáctica de las Ciencias, y viceversa", la pregunta por el sentido, por la buena enseñanza en términos éticos, debería comenzar a formar parte de la agenda de la didáctica de las ciencias naturales. Si bien falta aún un análisis exhaustivo al respecto, se observa que la didáctica de las ciencias está preocupada en la actualidad por tres temas principales, dos de los cuales muchas veces olvidan el objeto de estudio propio de la didáctica, que es la enseñanza. Estos temas son las ideas previas de alumnos y docentes, y la resolución de problemas. En general estos temas se plantean desde la lógica interna de las ciencias naturales y se pierde de vista el sentido educativo general de la investigación de estas temáticas y el sujeto principal del discurso didáctico, que es el docente.

3. La didáctica de las ciencias es una disciplina en gran medida autónoma, pero requiere del aporte de otras ciencias. Esto supone un trabajo muchas veces interdisciplinario que, coordinado por especialistas en el área, permita recuperar elementos de otras disciplinas desde la perspectiva propia del campo. La didáctica de las ciencias sigue (en muchos casos) monopolizada por los expertos disciplinares, que no suelen reconocer la especificidad del conocimiento didáctico y la necesidad de trabajar conjuntamente, para la construcción del conocimiento en el área, con pedagogos, psicólogos, lingüistas, sociólogos, epistemólogos.

4. Aparecen hoy nuevas visiones acerca de la ciencia, en particular en las ciencias naturales. Estas nuevas concepciones epistemológicas no tienen siempre, ni de modo inmediato, su correlato en las prácticas de la investigación, así como tampoco en las prácticas de la enseñanza, por lo que aún muchos investigadores siguen trabajando dentro de metodologías tradicionales, cercanas a la visión positivista de la ciencias naturales y sin conectar con las tradiciones interpretativas o críticas de las ciencias sociales. Esta situación se transfiere a la didáctica de las ciencias, que genera sus propios estándares de cientificidad, y a la propia enseñanza de las ciencias, que cristaliza modelos de ciencia autoritarios y dogmáticos heredados del positivismo.

5. El surgimiento de la teoría del caos, en las ciencias naturales, parece acercar este campo de conocimiento a las ciencias sociales: se recupera la complejidad del mundo, objeto de estudio de la ciencia, y se plantea la necesidad de un nuevo abordaje con una metodología tendiente a lo transdisciplinar.

6. Esta particularidad que asume hoy la visión epistemológica de las ciencias naturales, sumada a la complejidad de la enseñanza como práctica humana y social, con las consecuentes dificultades que conlleva su estudio teórico, puede derivar en dos situaciones: 
a. Una mayor dificultad para el estudio de un objeto doblemente complejo: la enseñanza de las ciencias naturales, con el riesgo de simplificar ambos elementos o uno de ellos, como ocurre en muchos casos hasta ahora.

b. Un reconocimiento, por parte de los didactas especiales, de las dificultades que supone el estudio de un objeto tan complejo y por lo tanto, la necesidad de considerar nuevas modalidades de investigación y un trabajo crecientemente interdisciplinario.

7. La didáctica de las ciencias ha tenido menos dificultades que la propia didáctica general para afirmar su propia cientificidad, de modo que en aquella parece bastante clausurado un debate que aun permea a esta última. Esta situación un tanto paradojal se ha dado por la confluencia de diversos factores: la formación de base de los didactas de las ciencias, y los nuevos modelos de ciencia que, en la década del ' 90 , nos permiten una caracterización más amplia y menos rígida de la investigación científica.

Creemos que es posible seguir profundizando en esta temática y encontrar muchas más aristas a la problemática. Sin embargo, dejamos abierta la puerta para reconstruir nuestras hipótesis y generar nuevas perspectivas sobre el tema.

\section{BIBLIOGRAFIA}

Adúriz-Bravo, A. (1999). Elementos de teoría y de campo para la construcción de un análisis epistemológico de la didáctica de las ciencias. Tesis de maestría: Universitat Autónoma de Barcelona.

Apostel, L. y otros (1977). La explicación en las ciencias. Barcelona: Martínez Roca.

Brown. H. J. (1984). La nueva filosofía de la ciencia. Madrid: Tecnos.

Camilloni, A. (1994). Epistemología de la didáctica de las ciencias sociales, en Aisenberg, B. y Alderoqui, 8. (comps). Didáctica de las ciencias sociales. Buenos Aires: Paidós.

Camilloni, A. el al. (1996). Corrientes didácticas contemporáneas. Buenos Aires: Paidós.

Carr, W. (1990). Hacia una ciencia crítica de la educación. Barcelona: Laertes.

Davini, C. (1996). Conflictos en la evolución de la didáctica. La demarcación de la didáctica general y de las didácticas especiales, en Camilloni, A. el al. Corrientes didácticas contemporáneas. Buenos Aires: Paidós.

Díaz Barriga, Á. (1997). La explicación científica. Una polémica desde la teoría del conocimiento, en Hoyos Medina, C. (coord.). Epistemología y objeto pedagógico. ¿Es la pedagogía una ciencia? México: CESU, UNAM, Plaza y Valdés.

Eder, ML. (1998). La Didáctica General y la Didáctica de las Ciencias Naturales. Una mirada epistemológica. Universidad de Buenos Aires.

Estany, A. (1993). Introducción a la filosofía de la ciencia. Barcelona: Crítica.

Fernández Rañada, A. (1990). Orden y caos. Barcelona: Prensa Científica. 
Gardner, H. y Boix-Mansilla, V. (1994). Teaching ter understanding in the disciplines and beyond. Teachers College Record, 96(2).

Giere, R. (1992). La explicación de la ciencia. Un acercamiento cognoscitivo. México: Consejo Nacional de Ciencia y Tecnología.

Gil-Pérez, 0. (1994). Diez años de investigación en didáctica de las ciencias: realizaciones y perspectivas. Enseñanza de las Ciencias, 12(2), 154-1 64.

Habermas, J. (1988). La lógica de las ciencias sociales. Madrid: Tecnos.

Hesse, M. (1980). Revolutions and reconstructions in the philosophy of science. Brighten: Harvester.

Hoyos Medina, C. (1997). Epistemología y discurso pedagógico. Razón y aporía en el proyecto de modernidad, en C. Hoyos Medina (coord.). Epistemología y objeto pedagógico. ¿Es la pedagogía una ciencia? México: CESU, UNAM, Plaza y Valdés.

Izquierdo, M. y Adúriz-Bravo, A. (en prensa). Epistemological foundations of school science. Science \& Education.

Jiménez Aleixandre, M. P. (1988). Enseñanza de las ciencias. Cuadernos de Pedagogía, 155, 8-10.

Laudan, L. (1986). El progreso y sus problemas. Hacia una teoría del crecimiento científico. Madrid: Ediciones Encuentro.

Luffiego, M., Bastida, MF., Ramos, F. y Soto, J. (1994). Epistemología, caos y enseñanza de las ciencias. Enseñanza de las Ciencias, 12.

Maggio, M. (1998). Aperturas en el marco de una nueva agenda para la didáctica: la perspectiva epistemológica como dimensión de análisis de las prácticas de la enseñanza. Tesis de maestría: Universidad de Buenos Aires.

Mata García, V. (1997). La determinación del pensamiento hipotético-deductivo en la construcción del conocimiento, en C. Hoyos Medina (coord.). Epistemología y objeto pedagógico. ¿ Es la pedagogía una ciencia? México: CESU, UNAM, PlazayValdés.

Morin, E. (1984). Ciencia con consciencia. Barcelona: Antrophos.

Pérez Tamayo, R. (1993). ¿Existe el método científico? Historia y realidad. Buenos Aires: Fondo de Cultura Económica.

Piaget, J. (1972). Epistemología de las ciencias humanas. Buenos Aires: Proteo.

Pla i Molins, M. (1993). Currículo y educación. Campo semántico de la didáctica. Universitat de Barcelona.

Popkewitz, T. (1994). Sociología política de las reformas educativas. Madrid: Morata.

Popper, K. (1973). La lógica de las Ciencias Sociales, en Adorno, T. et al. La disputa del positivismo en la sociología alemana. Barcelona: Grijalbo. 
Porlán, R. (1992). La Didáctica de las Ciencias. Una disciplina emergente. Cuadernos de Pedagogía, 210, 68-71.

Porlán, R. (1993). Constructivismo y escuela. Sevilla: Díada.

Porlán, R. (1998). Pasado, presente y futuro de la didáctica de las ciencias. Enseñanza de las Ciencias, 16(1), 175-185.

Prigogine, I.yStengers, I. (1983). La nueva alianza. Metamorfosis de la ciencia. Madrid: Alianza.

Rivera Medero, A. (1997). El debate epistemológico: una necesidad del proceso de investigación social, en C. Hoyos Medina (coord.). Epistemología y objeto pedagógico. ¿Es la pedagogía una ciencia? México: CESU, UNAM, Plaza y Valdés. 